\section{Brain perfusion patterns: Consistency and similarity with fMRI resting state networks and arterial vascular territories}

\author{
Alice Pirastru, ${ }^{1}$ Laura Pelizzari, ${ }^{1}$ \\ Niels Bergsland, 1,2 Mario Clerici, 1,3 \\ Pietro Cecconi, ${ }^{1}$ Raffaello Nemni, ${ }^{1,3}$ \\ Francesca Baglio, ${ }^{1}$ \\ Maria Marcella Laganà ${ }^{1}$ \\ ${ }^{1}$ IRCCS, Fondazione Don Carlo \\ Gnocchi, Milano, Italy; ${ }^{2}$ Buffalo \\ Neuroimaging Analysis Center, \\ Department of Neurology, Jacobs School \\ of Medicine and Biomedical Sciences, \\ University at Buffalo, State University of \\ New York, Buffalo, NY, USA; \\ ${ }^{3}$ Department of Physiopathology and \\ Transplants, University of Milano, \\ Milano, Italy
}

\section{Background}

Independent component analysis (ICA) is a well-known data driven technique used to extract patterns of common variation both from resting-state functional MRI (rsfMRI) and arterial spin labeling MRI (ASL) data. ${ }^{2}$ Our study aimed to: i) assess the consistency of ASL-derived cerebral blood flow (CBF) components (CBF-ICs) in healthy individuals (HI); ii) assess the similarity between the CBF-ICs and the spatial patterns of resting-state networks (RSN) and cerebral vascular territories (VT). ${ }^{3}$

\section{Methods}

ASL and rs-fMRI data were acquired for $92 \mathrm{HI}$ (median age [range] $=36.8[13-80]$ years, 45 females) to derive CBF-ICs and
RSN respectively. The images were preprocessed, then ICA was performed. Specifically, to test the CBF-ICs consistency, ICA (dimensionality $=15$ ) was run independently on two HI sub-groups (consisting of $46 \mathrm{HI}$ each). To investigate the CBF-ICs similarity to RSN and VT atlas, ICA (dimensionality $=20$ ) was performed on the whole HI group data. The Dice Similarity index (DSI) was computed to quantify both the CBF-ICs consistency and the CBF- ICs similarity to RSN and VT (slight $>0.1$, moderate $>0.2$, substantial $>0.4$, almost perfect $>0.6$ ).

\section{Results}

Moderate to substantial consistency was found for CBF-ICs in the occipital and frontal lobes, thalamus and cerebellum. The similarity between CBF-ICs and RSN was moderate for fronto-parietal, salience and sensori-motor networks and substantial for visual ones. Default mode network (DMN) constitutive nodes were split into three different CBF-ICs. The overlap between CBFICs and VT was slight to moderate for the anterior and posterior cerebral arteries respectively, and substantial for the medial ones.

\section{Conclusions}

ICA performed on CBF maps allows to consistently identifying common spatial patterns in HI. Although ASL and rs-fMRI are both hemodinamically-driven techniques, CBF-ICs and RSN provide complementary information. Furthermore CBF-ICs showed a lateralization similar to VT atlas (i.e. split left/right hemispheres). Therefore, CBF-ICs mirror both the vascular anatomical segregation and the local functional demand.
Correspondence: Alice Pirastru, IRCCS, Fondazione Don Carlo Gnocchi, Milano, Italy. E-mail: apirastru@dongnocchi.it

Key words: Neurovascular diseases; meeting.

Conference presentation: $9^{\text {th }}$ Annual Meeting of the International Society of Neurovascular Disease (ISNVD), May $30^{\text {th }}-31^{\text {st }}, 2019$, Ferrara, Italy.

This work is licensed under a Creative Commons Attribution 4.0 License (by-nc 4.0).

CO Copyright: the Author(s), 2019

Licensee PAGEPress, Italy

Veins and Lymphatics 2019; 8:8444

doi:10.4081/vl.2019.8444

\section{References}

1. Calhoun VD, Adali T, Pearlson GD, Pekar JJ. A method for making group inferences from functional MRI data using independent component analysis. Hum Brain Mapp 2001;14:140-51.

2. Dai W, Varma G, Scheidegger R, Alsop DC. Quantifying fluctuations of resting state networks using arterial spin labeling perfusion MRI. J Cereb Blood Flow Metab 2016;36:463-73.

3. 3. Paiva FF, Tannús A, Talagala SL, Silva AC. Arterial spin labeling of cerebral perfusion territories using a separate labeling coil. J Magn Reson Imaging 2008;27:970-7. 